DOI 10. 18307/2018. 0123

(C) 2018 by Journal of Lake Sciences

\title{
中全新世以来呼伦湖沉积物碳埋藏及其影响因素分析”
}

\author{
张风菊 ${ }^{1,2}$, 薛 滨 $^{1 * *}$, 姚书春 ${ }^{1}$ \\ (1: 中国科学院南京地理与湖泊研究所湖泊与环境国家重点实验室,南京 210008) \\ (2: 中国科学院大学,北京 100049)
}

\begin{abstract}
摘 要: 通过对内蒙古高原呼伦湖沉积物样品总有机碳含量 (TOC) 及其稳定同位素 $\left(\delta^{13} \mathrm{C}_{\text {org }}\right.$ )、总氮含量 $(\mathrm{TN})$ 和 TOC/TN $(\mathrm{C} / \mathrm{N})$ 值的测定, 结合沉积岩芯 $\mathrm{AMS}^{14} \mathrm{C}$ 年代标尺, 分析了中全新世以来呼伦湖沉积物有机碳埋藏速率随时间变化的趋 势及有机质的来源, 并探讨了影响呼伦湖有机碳埋藏的主要因素. 结果表明, 中全新世以来呼伦湖有机碳埋藏速率平均 值约为 $2.06 \mathrm{~g} /\left(\mathrm{m}^{2} \cdot \mathrm{a}\right)$, 碳储量约为 $35.25 \mathrm{Tg} \mathrm{C}$, 且总体上呈现增加趋势. 呼伦湖沉积物中有机质主要来源于外源输人, 但 近 $1000 \mathrm{a}$ 以来内源输入逐渐增加并占据优势. 呼伦湖有机碳埋藏速率与温度和降水均呈负相关, 表明在长时间尺度上, 升温及降水量的增加可能对呼伦湖的碳埋藏起到一定的抑制作用.
\end{abstract}

关键词: 碳储量;温度;降水; 总有机碳;稳定同位素;呼伦湖

\section{Organic carbon burial and its driving mechanism in the sediment of Lake Hulun, north- eastern Inner Mongolia, since the mid-Holocene}

\author{
ZHANG Fengju ${ }^{1,2}$, XUE Bin $^{1 * *}$ \& YAO Shuchun ${ }^{1}$ \\ (1: State Key Laboratory of Lake Science and Environment, Nanjing Institute of Geography and Limnology, Chinese Academy \\ of Sciences, Nanjing 210008, P.R.China) \\ (2: University of Chinese Academy of Sciences, Beijing 100049, P.R.China)
}

\begin{abstract}
A sediment core (HL16) was collected in the central part of Lake Hulun in northeastern Inner Mongolia using a piston core sampler. The total organic carbon ( TOC) content and its stable isotope $\left(\delta^{13} \mathrm{C}_{\text {org }}\right)$, total nitrogen ( TN) content and the total organic carbon and nitrogen $(\mathrm{C} / \mathrm{N})$ ratio of sediments were analyzed to investigate the temporal changes of organic carbon burial and its driving factors, as well as the organic carbon (OC) sources over the mid-Holocene. The OC burial rate presented an overall increasing trend for the last 7400 years, and the average OC burial rate was about $2.06 \mathrm{~g} /\left(\mathrm{m}^{2} \cdot \mathrm{a}\right)$, with a total C pool of 35.25 Tg C. Relative contributions of allochthonous and autochthonous OC input were estimated using a binary model, suggesting that OC being buried in Lake Hulun was mainly generated from terrigenous vegetation. The binary model also revealed that autochthonous OC demonstrated an increasing trend in the last 1000 years. The OC burial rate was negatively linked to temperature and precipitation based on Pearson analysis, implying that the increase in temperature and precipitation might lead to the decrease of OC burial in Lake Hulun in the long term scale.
\end{abstract}

Keywords: Carbon storage; temperature; precipitation; total organic carbon; stable isotope; Lake Hulun

随着全球气候变暖及碳排放问题的日益突出, 碳循环的研究越来越受到人们关注 ${ }^{[1-3]}$. 由于湖泊水体占 地球表面积较小 (仅占地球表面积的 $2 \%$ ), 早期研究往往忽视湖泊在碳循环中的作用,但近期越来越多的研 究证实湖泊中碳埋藏量非常丰富 ${ }^{[4-15]}$, 在全球碳循环中发挥着重要的作用 ${ }^{[6,9]}$. 开展湖泊碳埋藏的研究, 不 仅有助于了解湖泊生态系统碳循环规律及其影响机理, 而且对区域或全球碳循环规律的研究也具有重要的

* 国家自然科学基金项目(41573129、41372185)、科技基础性工作专项 (2014FY110400) 和湖泊与环境国家重点实验 室开放研究基金项目 (2015SKL015) 联合资助. 2017-02-13 收稿; 2017-04-04 收修改稿. 张风菊( 1988 ), 女, 博士研究生; E-mail: fjzhang8899@163.com.

** 通信作者; E-mail: bxue@ niglas.ac.cn. 
意义 ${ }^{[9]}$.

全新世是距今最近的地质记录年代, 也是研究程度相对高的一个年代. 许多学者致力于全新世以来湖 泊碳埋藏的研究 ${ }^{[10-15]}$, 目的是更好地了解古气候情境下的湖泊碳埋藏机制, 以便更好地预测未来气候变化 下湖泊的碳埋藏潜力. 中国湖泊数量众多, 类型多样, 分布广泛, 湖泊在碳循环中起着重要的作用 ${ }^{[15-21]}$. 有关 国内湖泊碳埋藏方面已经开展了不少研究, 如在长江中下游湖泊 ${ }^{[16-17]}$ 、青海湖 ${ }^{[18]}$ 及新疆博斯腾湖 ${ }^{[19]}$ 等开展 了碳埋藏及其影响因素的研究, 但这些研究往往限于近代或现代湖泊碳埋藏, 有关全新世以来湖泊碳埋藏 的研究相对较少. Wang 等 ${ }^{[15]}$ 曾综述了全新世以来中国 58 个湖泊的碳埋藏,但文中是以 $1000 \mathrm{a}$ 作为间隔进 行分析, 时间精度相对较低, 此外, 在研究气候变化对有机碳埋藏的影响时, 文中采用的是现代气候参数, 而 没有考虑历史时期气候条件的变化. 因此,迫切需要开展中国湖泊长时间尺度、高精度的碳埋藏研究.

呼伦湖是我国北方干旱一半干旱区面积最大的浅水湖泊, 对气候、环境的变化反应敏感, 在干旱一半干 旱区水体中具有代表性. 本文以钻取自呼伦湖盆中心 (图 1) 的一套长度为 $125 \mathrm{~cm}$ 的沉积岩芯为研究对象, 开展了中全新世以来呼伦湖沉积物有机碳埋藏的时间变化趋势、碳来源以及影响因素的研究, 以期进一步 理解干旱一半干旱区大型浅水湖泊碳循环过程与特征, 并为与湿润区湖泊碳埋藏的对比研究积累一定的基 础资料.

\section{1 区域地理概况}

呼伦湖 ( 又名呼伦池、达责诺尔, $48.55^{\circ} \sim 49.33^{\circ} \mathrm{N}, 116.97^{\circ} \sim 117.81^{\circ} \mathrm{E}$ ) 是我国第五大湖, 也是我国北方 第一大湖. 位于呼伦贝尔草原西部新巴尔虎左旗、新巴尔虎右旗和满洲里市之间. 当湖水位在 $545.3 \mathrm{~m}$ 时, 蓄水量约 138 亿 $\mathrm{m}^{3}$, 水面面积约 $2339 \mathrm{~km}^{2}$, 最大水深 $8 \mathrm{~m}$, 平均水深 $5.7 \mathrm{~m}$. 湖面呈不规则的斜长型, 湖面长 $93 \mathrm{~km}$, 最大宽度 $41 \mathrm{~km}$, 平均宽度 $25 \mathrm{~km}$. 湖周长 $447 \mathrm{~km}$. 呼伦湖集水面积为 $153669 \mathrm{~km}^{2}$, 其中我国境内部分 为 $37214 \mathrm{~km}^{2}$, 蒙古人民共和国为 $116455 \mathrm{~km}^{2[22]}$ (图 1).

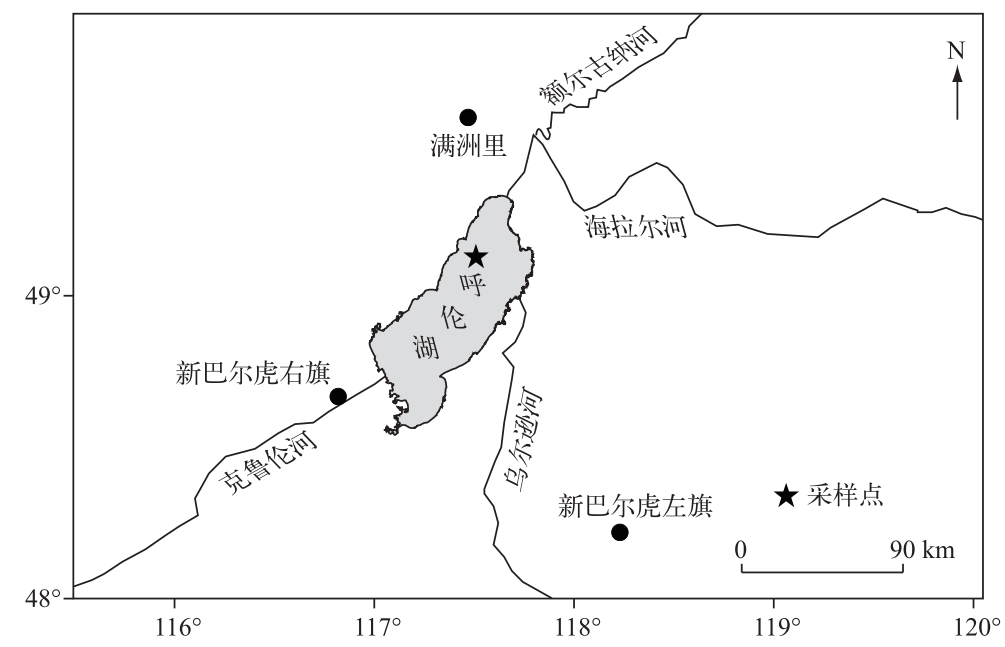

图 1 呼伦湖及采样点 (星号表示) 位置

Fig.1 Map of Lake Hulun showing the location of the HL16 sediment core (the star)

呼伦湖地区属中温带大陆性气候. 冬季严寒漫长, 夏季温凉短促, 积雪封冻期 6 个月左右, 最大冰层厚 $1.30 \mathrm{~m}$. 湖区多年平均气温 $-0.5 \sim 0.5^{\circ} \mathrm{C}$, 年平均降水量 $285 \mathrm{~mm}$, 蒸发量 $1650 \sim 1700 \mathrm{~mm}$, 全年盛行西北风, 多 年平均风速 $4.2 \mathrm{~m} / \mathrm{s}$. 呼伦湖主要补给水源为源于蒙古国东部的克鲁伦河、连接呼伦湖和贝尔湖的乌尔逊河 以及海拉尔河, 排泄河流为中俄界河一额尔古纳河, 并经额尔古纳河外流人鄂霍茨克海. 湖区东北部的新开 河为吞吐性河流,高水位时,海拉尔河通过新开河补给呼伦湖 ${ }^{[23]}$.

呼伦湖流域及湖周主要土壤类型为栗钙土、草甸土及部分盐化/碱化草甸土 ${ }^{[23]}$. 湖泊地域植被为草原, 
C3 植物如大针茅 (Stipa grandis P. Smirn.) 、羊草 (Leymus chinemis ( Trin.) Tzvel.) 是呼伦贝尔草原植物群落优 势种 ${ }^{[24]}$. 经采样调查, 呼伦湖共有浮游植物 142 种属, 以绿藻、蓝藻和硅藻占优势, 且浮游植物平均生物量为 $6.995 \mathrm{mg} / \mathrm{L}^{[25]}$.

\section{2 样品采集与分析方法}

\section{1 样品采集}

2016 年 3 月,在呼伦湖中心 $\left(49.13^{\circ} \mathrm{N}, 117.51^{\circ} \mathrm{E}\right)$ 水深 $6.4 \mathrm{~m}$ 处进行冰上钻探, 获取一长度为 $125 \mathrm{~cm}$ 的 沉积岩芯 (命名为 HL16), 现场对岩芯进行拍照与岩性描述, 岩芯带回实验室后进行对剖, 并按照 $1 \mathrm{~cm}$ 间距 切分岩芯, 得到 125 个湖泊沉积样品用于沉积物分析.

根据岩性变化可将岩芯分为 3 段:上部 $(0 \sim 35 \mathrm{~cm})$ 为黑灰色淤泥质粉砂沉积, 间或含少量粗砂; 中间部 分 (35 91 cm) 为黑灰色泥质粉砂沉积, 沉积物颗粒相对较细, 粗砂含量较少; 底部 $(91 \sim 125 \mathrm{~cm})$ 为浅灰色砂 质沉积物, 颗粒相对较粗, 见较多粗砂.

\section{2 样品分析}

2.2.1 $\mathrm{AMS}^{14} \mathrm{C}$ 年龄测定 从 HL16 岩芯样品中, 分别选取岩芯顶部 $(0 \sim 1 \mathrm{~cm}) 、$ 中部 $(50 \sim 51 \mathrm{~cm})$ 及底部 $(119 \sim 120 \mathrm{~cm})$ 共 3 个富含有机质的全岩样品, 寄往美国 Beta 实验室进行 $\mathrm{AMS}^{14} \mathrm{C}$ 定年. 测年所用 ${ }^{14} \mathrm{C}$ 半衰期 为 $5568 \mathrm{a}, \mathrm{BP}$ 为距 1950 年的年代. 同时所测定的全部 ${ }^{14} \mathrm{C}$ 年代通过 Calib 7.10 校正软件中的 Intcal 13 树轮校 正曲线进行校正以获得日历年 (单位为 $\mathrm{cal} \mathrm{a} \mathrm{BP})^{[26]}$. 根据测年层位 $2 \sigma$ 误差范围内校正年龄的均值, 线性内 插计算得到钻孔岩芯样品的年龄. 定年材料为样品中的有机碳, 具体方法见 Nakamura 等 $^{[27]}$ 的描述.

2.2 .2 有机碳 ( TOC ) 、总氮 ( TN ) 及有机质碳同位素 $\left(\delta^{13} \mathrm{C}_{\mathrm{org}}\right)$ 含量的测定 将真空冷冻干燥后的样品研磨成 80 目左右的粉末, 称取适量样品加人 $10 \%$ 的 $\mathrm{HCl}$, 多次搅拌直至没有气泡冒出, 以除去样品中的碳酸盐; 然 后反复用蒸馏水清洗离心至中性; 低温 $\left(40^{\circ} \mathrm{C}\right)$ 烘干后研磨至 80 目. 称取适量处理后的样品置于小锡杯中, 用美国 EAI 公司生产的 EA-3000 型元素分析仪测定样品中的 TOC 和 TN 含量, 通过每隔 8 个样品插人标样 的方法来控制测试结果的质量, 测试误差 $<0.1 \%$. TOC/TN 比值 ( $\mathrm{C} / \mathrm{N}$ 值) 即为其摩尔比. $\delta^{13} \mathrm{C}_{\mathrm{org}}$ 根据有机碳 含量称取适量样品, 通过 Delta Plus 同位素质谱仪测定, 分析精密度优于 $0.2 \%$, 结果以 VPDB 标准表示. 所 有测试均在中国科学院南京地理与湖泊研究所湖泊与环境国家重点实验室内完成.

2.2.3 有机碳埋藏速率的计算 呼伦湖沉积物有机碳埋藏速率 $\left(O C B R, \mathrm{~g} /\left(\mathrm{m}^{2} \cdot \mathrm{a}\right)\right)$ 由沉积物 TOC 含量 $(\%) 、$ 沉积速率 $(S R, \mathrm{~cm} / \mathrm{a})$ 及干物质密度 $\left(D B D, \mathrm{~g} / \mathrm{cm}^{3}\right)$ 计算得到. $S R$ 由沉积物深度一年代模式得到, $D B D$ 为沉积物样品湿重 $\left(M_{\mathrm{w}}\right)$ 和干重 $\left(M_{\mathrm{d}}\right)$ 之差与样品干重体积 $\left(V, \mathrm{~cm}^{3}\right)$ 之比, 计算公式分别为:

$$
\begin{gathered}
O C B R=T O C \cdot S R \cdot D B D \\
I C B R=T I C \cdot S R \cdot D B D \\
D B D=\left(M_{\mathrm{w}}-M_{\mathrm{d}}\right) / V
\end{gathered}
$$

\section{3 结果与分析}

\section{1 年代序列的建立}

岩芯 $0 \sim 1 \mathrm{~cm} 、 50 \sim 51 \mathrm{~cm}$ 及 $119 \sim 120 \mathrm{~cm}$ 深度处样品的 $\mathrm{AMS}^{14} \mathrm{C}$ 年龄如表 1 所示. 由于湖泊沉积物中全 有机质来源较为复杂, 易受到生物体利用无机碳所导致的 “碳库效应” 的影响而使测量年龄偏老, 因此还必 须进行 “碳库” 校正. 呼伦湖岩芯顶部 $0 \sim 1 \mathrm{~cm}$ 的测年结果约为 $800 \mathrm{a}$,这一数据可以近似看作是 “碳库效应” 对呼伦湖沉积物年龄的影响, 假设在整个钻孔沉积中碳库效应的影响保持不变, 首先将全部原始 $\mathrm{AMS}^{14} \mathrm{C}$ 年 龄减去 800 a 以消除 “碳库效应” 的影响, 然后将得到的年龄进行校正以获得日历年. 将本文的深度一年代结 果与 Xiao 等 ${ }^{[28]}$ 在同一地点钻取的岩芯深度一年代结果进行比较, 发现两个岩芯深度一年代模式相对一致 (图 2), 因此, 认为 HL16 钻孔的年代结果较为可靠. HL16 钻孔的年龄一深度模型显示, 钻孔沉积底部 125 $\mathrm{cm}$ 处样品的沉积年代可追溯至中全新世.

此外, 我们曾于 2011 年 12 月在 HL16 岩芯钻取位置取得一短柱状岩芯 (HL2011 孔, 岩芯长度 $74 \mathrm{~cm}$ ), 并采用 ${ }^{210} \mathrm{~Pb}$ 和 ${ }^{137} \mathrm{Cs}$ 测年方法对该短岩芯进行了定年, 具体年代结果可参考桂智凡 ${ }^{[29]}$. 将 $\mathrm{HL} 16$ 岩芯 $\mathrm{AMS}^{14} \mathrm{C}$ 
表 1 呼伦湖 $\mathrm{HL} 16$ 岩芯样品的 $\mathrm{AMS}^{14} \mathrm{C}$ 年代

Tab.1 AMS radiocarbon dates of samples from HL16 sediment core in Lake Hulun

\begin{tabular}{cccccc}
\hline 实验室编号 & 样品深度 $/ \mathrm{cm}$ & 测年材料 & $\delta^{13} \mathrm{C} / \% 0$ & $\mathrm{AMS}^{14} \mathrm{C}$ 年代/a BP & 校正后的年代 $(2 \sigma) / \mathrm{cal} \mathrm{a} \mathrm{BP}$ \\
\hline Beta-437852 & $0 \sim 1$ & 有机质 & -27.2 & $800 \pm 30$ & $0-10$ \\
Beta-437853 & $50 \sim 51$ & 有机质 & -27.0 & $1900 \pm 31$ & $951-1063$ \\
Beta-437855 & $119 \sim 120$ & 有机质 & -26.5 & $6800 \pm 33$ & $6772-6927$ \\
\hline
\end{tabular}

年代结果与 HL2011 孔的 ${ }^{210} \mathrm{~Pb}$ 和 ${ }^{137} \mathrm{Cs}$ 年代结果进行对 比,发现两者的年代结果差异较大. 比如, HL2011 岩芯

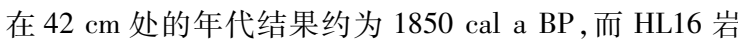

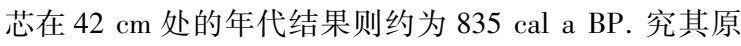
因,我们猜测可能是由于 HL16 岩芯存在沉积不连续的 现象. 一方面,从呼伦湖全新世以来不同时段的沉积速 率 (因为本研究的年代数据较少, 因此沉积速率的计算 采用年代相近的 Xiao 等 ${ }^{[28]}$ 的年代数据) 来看 (图 3), 该 岩芯顶部沉积速率较高, 而底部较低. 这可能是由于顶 部保存条件较好,沉积速率高,下部沉积物存在多次侵 蚀,仅有部分保存下来,造成该时期的沉积层较薄,因而 沉积速率较低.

此外, 呼伦湖作为地处西风与东亚季风交汇区的吞吐 性湖泊,湖水较浅且湖区风浪较大 (平均风速 $4.2 \mathrm{~m} / \mathrm{s}$ ), 很 容易在风浪扰动下造成沉积物的侵蚀. 同时, 呼伦湖多 年水量平衡关系研究表明,乌尔逊河多年平均径流量为 6.9 亿 $\mathrm{m}^{3}$, 克鲁伦河多年平均径流量为 4.9 亿 $\mathrm{m}^{3}$, 新开 河出湖径流量为 1.75 亿 $\mathrm{m}^{3}$, 湖泊人湖及出湖径流也有 可能导致沉积物的侵蚀及搬运. 最后, HL16 岩芯粒度 结果 (图 4) 表明,沉积岩芯中出现多次粒径变大、颗粒 变粗的现象,可能代表当时湖泊收缩、湖水较浅的时 期 ${ }^{[28]}$, 在浅水强动力条件下, 也有可能导致沉积物的冲 刷侵蚀, 最终造成沉积地层的缺失.

综上所述, 呼伦湖 $\mathrm{AMS}^{14} \mathrm{C}$ 年代与 ${ }^{210} \mathrm{~Pb}$ 及 ${ }^{137} \mathrm{Cs}$ 年 代结果存在不一致的原因可能与呼伦湖存在的沉积不 连续有关. 因此, 本文所用年代均为 $\mathrm{AMS}^{14} \mathrm{C}$ 测年结果, 而不考虑 ${ }^{210} \mathrm{~Pb}$ 和 ${ }^{137} \mathrm{Cs}$ 的年代结果.

\section{2 沉积岩芯 TOC $、 \mathrm{C} / \mathrm{N}$ 比值及 $\delta^{13} \mathrm{C}_{\mathrm{org}}$ 的变化特征}

中全新世以来呼伦湖沉积物 TOC 含量差异显著 (图 5), 最小值为 $0.85 \%$, 最大值为 $3.14 \%$, 平均值为

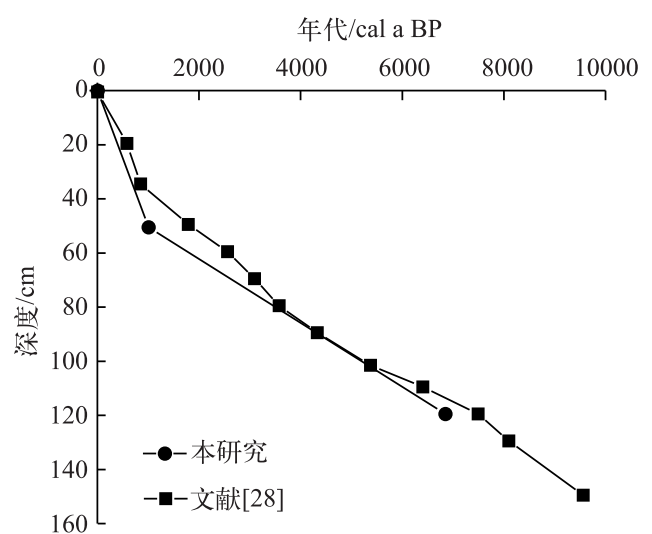

图 2 HL16 岩芯年代一深度模式及 与文献 $[28]$ 结果的比较

Fig.2 Lithology and age depth model of the HL16 sediment core and comparison with reference[28]

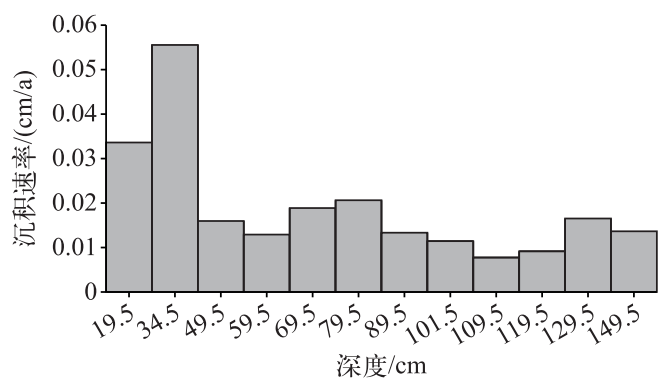

图 3 HL06 岩芯沉积速率的变化 (数据源于文献 $[28]$ )

Fig. 3 The sediment rate of HL06 core

(data were from reference[28])

$1.71 \%$. 其中在 $125 \sim 75 \mathrm{~cm}$ (约为 7315-3080 cal a BP) TOC 含量呈现平稳增加的趋势, 从 $0.85 \%$ 增加至 $1.97 \%$; 在 75 63 cm (约为 3080-2065 cal a BP) TOC 含量快速下降至 $1.33 \%$; 在 63 35 cm (约为 2065-695 cal a BP) TOC 含量开始增加至剖面最大值 (3.14\%) ; 在 35 17 cm (约为 695-335 cal a BP) TOC 含量呈下降 趋势; 在 $17 \mathrm{~cm}$ 以上 (约为 $335 \mathrm{cal}$ a BP 以来) TOC 含量逐渐增加. 总体上来说, 呼伦湖 TOC 含量呈现增加趋 势. $\mathrm{C} / \mathrm{N}$ 值变化范围为 8.8 17.3, 平均值为 12.4 (图 5). 在 $125 \sim 91 \mathrm{~cm}$ (约为 $7315-4435 \mathrm{cal} \mathrm{a} \mathrm{BP}$ ) $\mathrm{C} / \mathrm{N}$ 值较 高, 且呈现增加趋势, 变化范围为 11.9 17.3; 在 91 51 cm (约为 4435-1050 cal a BP) $\mathrm{C} / \mathrm{N}$ 值相对较稳定, 变化范围为 11.9 13.7; 在 51 35 cm(约为 $1050-695 \mathrm{cal} \mathrm{a} \mathrm{BP}) \mathrm{C} / \mathrm{N}$ 值波动增加, 变化范围为 $10.3 \sim 14.7$; 在 

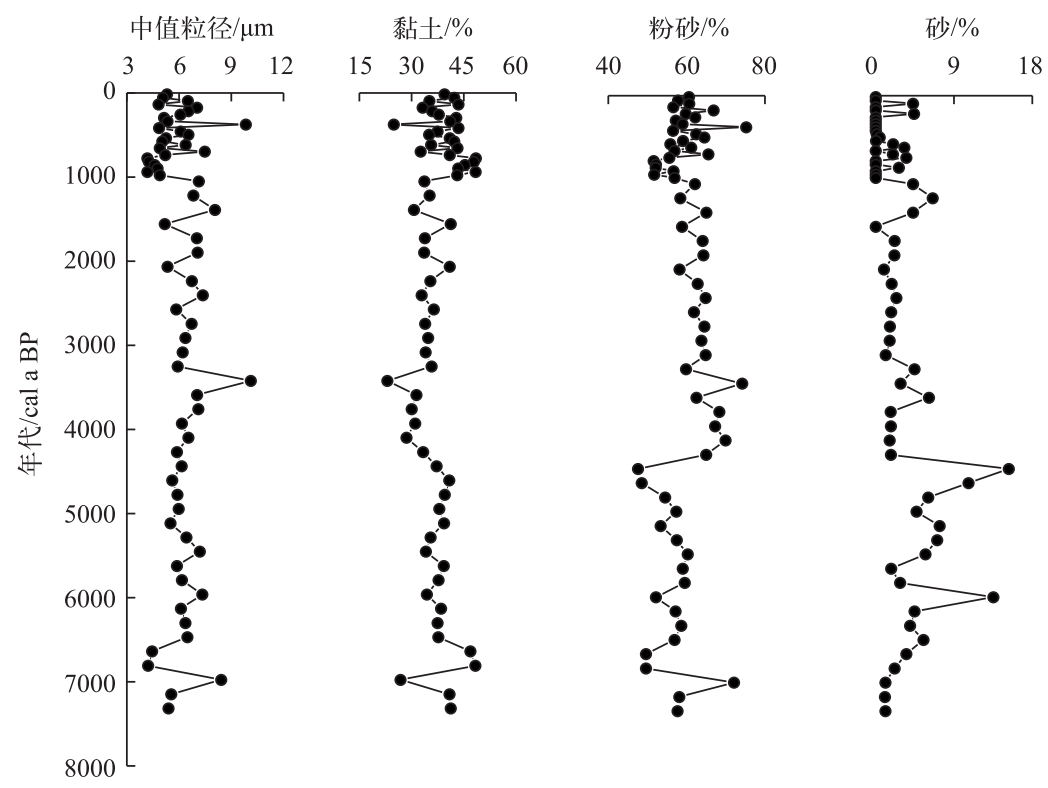

图 4 HL16 岩芯粒度分布

Fig.4 Distribution of grain size in HL16 sediment core

$35 \mathrm{~cm}$ 以上 (约为 $695 \mathrm{cal}$ a $\mathrm{BP}$ 以来) $\mathrm{C} / \mathrm{N}$ 值逐渐减小. 有机质 $\delta^{13} \mathrm{C}_{\mathrm{org}}$ 的变化范围为 $-27.92 \%{ }_{00} \sim-25.77 \%$ (图 5 ). 在 $125 \sim 91 \mathrm{~cm}$ (约为 7315-4435 cal a BP) $\delta^{13} \mathrm{C}_{\mathrm{org}}$ 存在显著的偏轻趋势, 变化范围为 $-27.74 \% 0 \sim-26.53 \%$; 在 $91 \sim 53 \mathrm{~cm}$ (约为 $4435-1220 \mathrm{cal} \mathrm{a} \mathrm{BP}$ ) $\delta^{13} \mathrm{C}_{\mathrm{org}}$ 呈现偏重趋势, 变化范围为 $-27.18 \%$ $-25.76 \%$; 在 $53 \mathrm{~cm} \mathrm{以}$

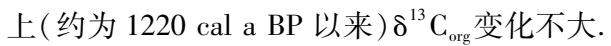

\section{3 沉积岩芯 $O C B R$ 变化特征}

中全新世以来呼伦湖 $O C B R$ 变化范围为 $1.00 \sim 5.39 \mathrm{~g} /\left(\mathrm{m}^{2} \cdot \mathrm{a}\right)$, 平均值为 $2.06 \mathrm{~g} /\left(\mathrm{m}^{2} \cdot \mathrm{a}\right)$ (图 5$)$. $O C B R$ 变化特征与 TOC 含量变化趋势相近, 整体上呈现增加趋势. 具体来说, 岩芯 $125 \sim 91 \mathrm{~cm}$ (约为 7315-4435 cal a BP ) $O C B R$ 表现为增加趋势, 从 $1.00 \mathrm{~g} /\left(\mathrm{m}^{2} \cdot \mathrm{a}\right)$ 增加至 $1.97 \mathrm{~g} /\left(\mathrm{m}^{2} \cdot \mathrm{a}\right) ; 91 \sim 53 \mathrm{~cm}$ (约为 4435-1220 cal a BP) OCBR 也呈增加趋势, 但变化幅度较小, 变化范围为 $1.30 \sim 1.83 \mathrm{~g} /\left(\mathrm{m}^{2} \cdot \mathrm{a}\right) ; 53 \sim 35 \mathrm{~cm}($ 约为 $1220-$ $696 \mathrm{cal} \mathrm{a} \mathrm{BP}) O C B R$ 呈现快速增加趋势, 并达到整个剖面最大值 $\left(5.39 \mathrm{~g} /\left(\mathrm{m}^{2} \cdot \mathrm{a}\right)\right) ; 35 \sim 17 \mathrm{~cm}(696 \sim 335 \mathrm{cal}$

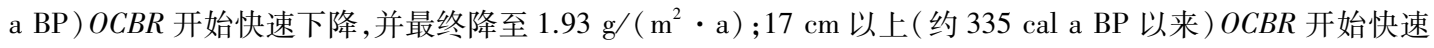
上升,并在岩芯顶部达到整个剖面的次高值 $\left(4.79 \mathrm{~g} /\left(\mathrm{m}^{2} \cdot \mathrm{a}\right)\right)$.

此外, 将中全新世以来呼伦湖 $O C B R$ 与呼伦湖面积及沉积年代相乘, 得到中全新世以来呼伦湖有机碳 储量为 $35.25 \mathrm{Tg} \mathrm{C}\left(1 \mathrm{Tg}=10^{12} \mathrm{~g}\right)$.

\section{4 讨论}

\section{1 呼伦湖有机碳来源分析}

湖泊沉积物中 TOC 主要有两大来源: 来自湖泊生物 (如浮游生物等) 的内源成因以及来自湖区周围人 湖径流带人的陆生生物的外源成因 ${ }^{[30]}$. 研究表明, 沉积物有机质的 $\mathrm{C} / \mathrm{N}$ 值可指示有机质的来源, 湖泊水生 植物由于含有较高的蛋白质和脂类, 其 $\mathrm{C} / \mathrm{N}$ 值一般在 $4 \sim 10$ 之间, 而陆生维管植物和挺水植物富含纤维素 和木质素, 其 $\mathrm{C} / \mathrm{N}$ 值一般大于 $20^{[31]}$. 呼伦湖沉积物 TOC 与 TN 含量的回归分析表明两者高度线性相关, 且 截距接近于 0 , 表明沉积物 TN 主要来源于有机质, 而黏土颗粒结合的铵态氮含量则可忽略不计 ${ }^{[32]}$, 因此, 呼 伦湖 $\mathrm{C} / \mathrm{N}$ 值可指示沉积物有机质的来源.

如前所述, 呼伦湖 $\mathrm{C} / \mathrm{N}$ 值变化范围为 8.8 17.3, 均值为 12.4(图 5), 表明呼伦湖沉积物中的内、外源有 

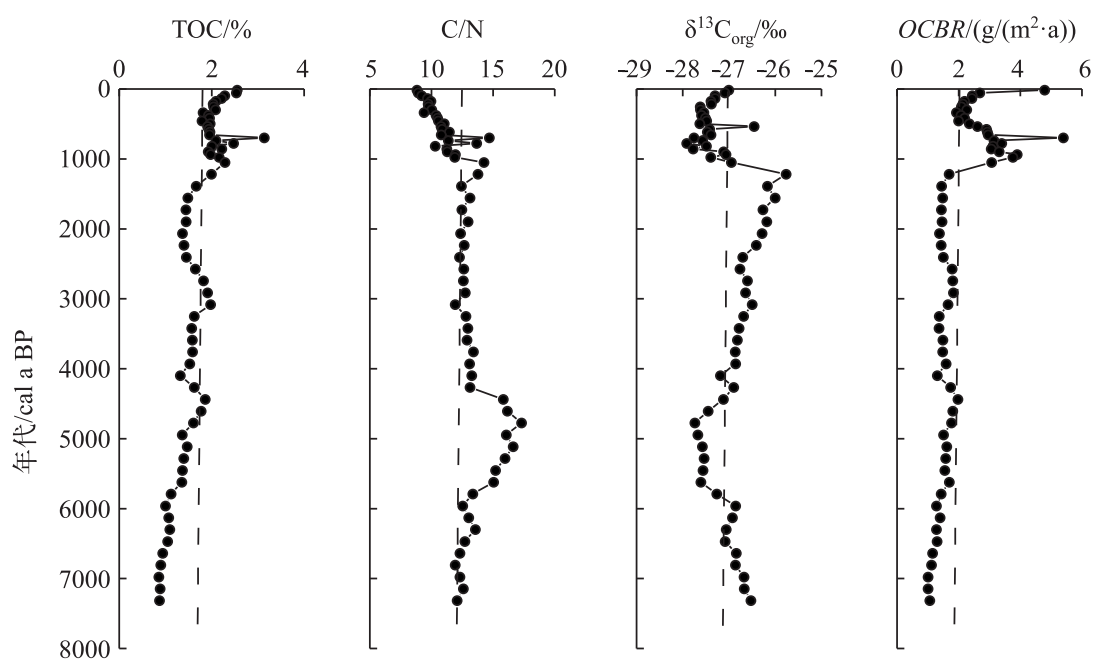

图 $5 \mathrm{HL} 16$ 岩芯 TOC、C/N、 $\delta^{13} \mathrm{C}_{\text {org }}$ 以及 $O C B R$ 随时间的变化 (图中虚线代表各指标平均值)

Fig.5 Temporal changes of TOC, C/N, $\delta^{13} \mathrm{C}_{\text {org }}$ and OCBR in HL16 sediment core (the dashed lines represent the average values of the indexes)

机质在沉积物有机质中各占有一定比例. 岩芯 $125 \sim 91 \mathrm{~cm} \mathrm{C} / \mathrm{N}$ 值相对较高, 最小值为 11.9 , 最大值为 17.3 , 且该最大值也为整个岩芯的最高值, 表明约在 7315-4435 cal a BP 时期呼伦湖陆源输人有机质含量相对较

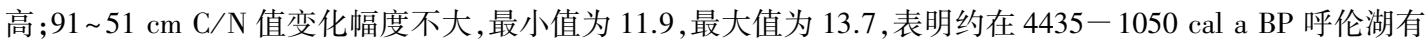
机质中外源输人的比例较前期有所下降, 内源有机碳含量增加; $51 \mathrm{~cm}$ 以上 $\mathrm{C} / \mathrm{N}$ 值波动减小, 且基本均小于 11.0 , 表明 $1050 \mathrm{cal}$ a BP 以来湖泊内源有机质所占比例逐渐增加且最终占据优势. 此外, 研究还表明呼伦湖 沉积物 $\mathrm{C} / \mathrm{N}$ 值与湖区土壤 $\mathrm{C} / \mathrm{N}$ 值 (约为 15.12$)^{[33]}$ 较为接近, 因此, 我们认为中全新世以来呼伦湖沉积物有 机质可能主要来源于陆源物质,但近千年来沉积物有机碳可能以内源为主.

湖泊沉积物中有机质 $\delta^{13} \mathrm{C}$ 与有机质来源紧密相关 ${ }^{[34]}$. 根据光合作用机理的不同, 陆生植物大致可分为 $\mathrm{C} 3$ 植物、C4 植物以及 CAM 植物 3 种类型. 总体上, C 3 植物的 $\delta^{13} \mathrm{C}$ 变化范围为 $-37 \% 0 \sim-24 \%, \mathrm{C} 4$ 植物的 $\delta^{13} \mathrm{C}$ 变化范围为 $-19 \% 0 \sim-9 \%$, CAM 植物的 $\delta^{13} \mathrm{C}$ 值为 $-30 \% 0 \sim-10 \%{ }^{[34]}$. 对于湖泊水生植物来说, 挺水植物 光合作用时一般直接利用大气中的 $\mathrm{CO}_{2}$ 进行光合作用, 其 $\delta^{13} \mathrm{C}$ 值 $(-30 \%$ - 24\% $)$ 与陆生 $\mathrm{C} 3$ 植物相近; 沉 水植物则由于主要吸收水中重碳酸盐溶解释放出的 $\mathrm{CO}_{2}$, 造成 $\delta^{13} \mathrm{C}$ 值相对偏正 ${ }^{[35]}$. 从呼伦湖有机质 $\delta^{13} \mathrm{C}$ 变 化范围来看 (图 5), 呼伦湖沉积物中有机质可能具有多源性. 但呼伦湖有机质 $\delta^{13} \mathrm{C}$ 值和湖周草地 $\left(-25.96 \%\right.$ ) 、土壤 $(-26.04 \% 0)$ 以及牲畜粪便 $(-26.13 \% 0)$ 的 $\delta^{13} \mathrm{C}$ 均较接近 ${ }^{[33]}$, 说明中全新世以来呼伦湖有机 质可能以陆源输人为主.

为定量研究陆源及内源有机质对呼伦湖沉积物有机质的相对贡献, 我们采用二元模式 (the binary model $)^{[36]}$ 估算了呼伦湖沉积物外源和内源有机质的相对输人量. 二元模式的计算公式为:

$$
\begin{gathered}
C(i)=C_{1}(i)+C_{\mathrm{u}}(i) \\
N(i)=N_{1}(i)+N_{\mathrm{u}}(i) \\
R_{1}(i)=C_{1}(i) / N_{1}(i) \\
R_{\mathrm{u}}(i)=C_{\mathrm{u}}(i) / N_{\mathrm{u}}(i)
\end{gathered}
$$

式中, $C(i)$ 和 $N(i)$ 分别为第 $i$ 层沉积物样品中的 TOC 和 TN 含量, $C_{1}(i)$ 和 $N_{1}(i)$ 分别为第 $i$ 层沉积物样品中 来自外源的 TOC 和 TN, $C_{\mathrm{u}}(i)$ 和 $N_{\mathrm{u}}(i)$ 为第 $i$ 层沉积物样品中来自内源的 TOC 和 TN, $R_{1}(i)$ 和 $R_{\mathrm{u}}(i)$ 分别为 深度为 $i$ 的沉积物中来自陆源和内源的碳氮比. 由上述几个方程式可得:

$$
\begin{aligned}
& N_{1}(i)=\left[C(i)-R_{\mathrm{u}}(i) \cdot N(i)\right] /\left[R_{1}(i)-R_{\mathrm{u}}(i)\right] \\
& N_{\mathrm{u}}(i)=\left[C(i)-R_{1}(i) \cdot N(i)\right] /\left[R_{\mathrm{u}}(i)-R_{1}(i)\right]
\end{aligned}
$$




$$
\begin{aligned}
& C_{1}(i)=R_{1}(i)\left[C(i)-R_{\mathrm{u}}(i) \cdot N(i)\right] /\left[R_{1}(i)-R_{\mathrm{u}}(i)\right] \\
& C_{\mathrm{u}}(i)=R_{\mathrm{u}}(i)\left[C(i)-R_{1}(i) \cdot N(i)\right] /\left[R_{\mathrm{u}}(i)-R_{\mathrm{l}}(i)\right]
\end{aligned}
$$

因此, 如果知道 $R_{1}(i)$ 和 $R_{\mathrm{u}}(i)$ 的值, 即可计算出不同深度沉积物内源和外源有机质所占的比例. 零级近 似可取 $R_{1}(i)=R_{1}$ 和 $R_{\mathrm{u}}(i)=R_{\mathrm{u}}$, 考虑到呼伦湖沉积物 $\mathrm{C} / \mathrm{N}$ 比值变化范围为 $8.8 \sim 17.3$, 我们把 $R_{1}$ 和 $R_{\mathrm{u}}$ 分别赋 值为 18 和 8 . 进而可得出不同时间段内源有机质和外源有机质对沉积物有机质的贡献.

研究结果表明, 呼伦湖沉积物有机碳来源以外源为主, 但近 $1000 \mathrm{a}$ 以来内源有机碳的比例开始增加, 并 逐渐占据优势 (图 6).

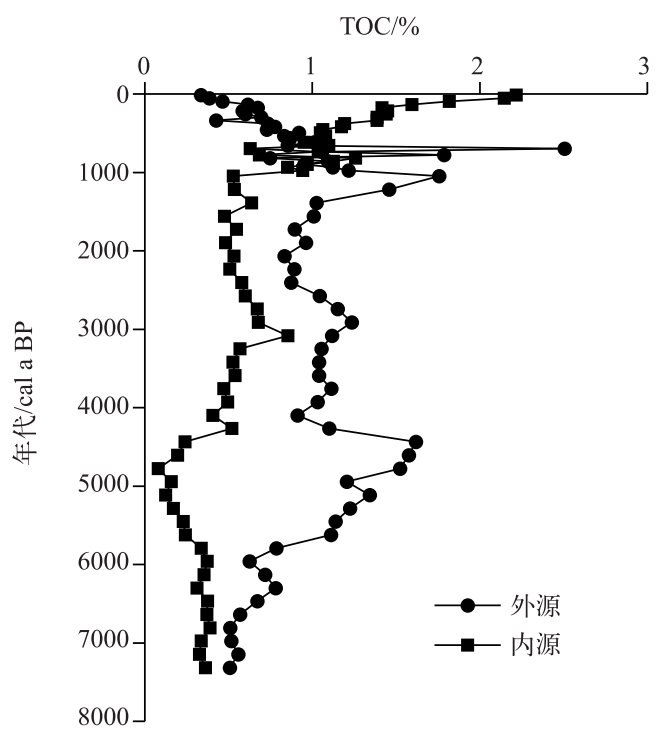

图 6 中全新世以来呼伦湖沉积物陆源及内源 TOC 的相对贡献

Fig.6 Variations of the allochthonous and autochthonous TOC in the HL16 sediment core from Lake Hulun

\section{2 与不同区域湖泊碳埋藏的对比}

中全新世以来呼伦湖 $O C B R$ 约为 $2.06 \mathrm{~g} /\left(\mathrm{m}^{2} \cdot \mathrm{a}\right)$, 和芬兰湖泊全新世以来的 $O C B R$ 相近 $\left(1.8 \mathrm{~g} /\left(\mathrm{m}^{2}\right.\right.$. a) $)^{[14]}$, 但低于格陵兰西南部湖泊全新世以来的 $O C B R\left(5 \mathrm{~g} /\left(\mathrm{m}^{2} \cdot \mathrm{a}\right)\right)^{[10]}$ 、欧洲湖泊长时间尺度的 $O C B R(5.6$ $\left.\mathrm{g} /\left(\mathrm{m}^{2} \cdot \mathrm{a}\right)\right)^{[13]}$ 以及加拿大 Alberta 地区湖泊全新世以来的 $O C B R\left(15 \mathrm{~g} /\left(\mathrm{m}^{2} \cdot \mathrm{a}\right)\right)^{[11]}$, 也远低于呼伦湖全新 世大暖期及现代的 $O C B R^{[37]}$. 一方面, 这可能与呼伦湖地处干早一半干旱的高纬度地区, 地表径流相对贫乏, 流域植被生长季节较短以及较低的初级生产力有关; 另一方面,这也和呼伦湖较低的沉积速率相关.

\section{3 碳埋藏影响因素分析}

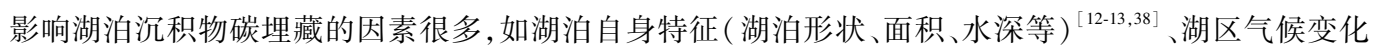

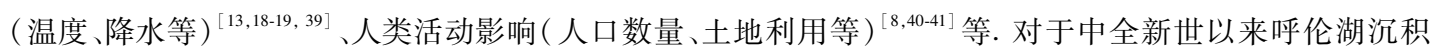
物碳埋藏的影响因素来说, 考虑到人类活动在中全新世期间相对较弱, 气候变化可能是控制呼伦湖碳埋藏 的主要因子. 因此, 我们在此只考虑气候因素中的温度和降水对呼伦湖中全新世以来碳埋藏的可能影响.

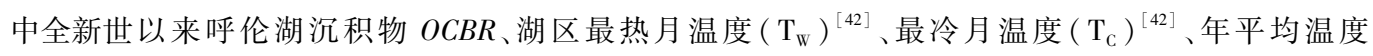
$\left(\mathrm{T}_{\mathrm{A}}\right)^{[42]}$ 及年均降水量 $\left(\mathrm{P}_{\mathrm{A}}\right)^{[42]}$ 随时间的变化趋势如图 7 所示. 由图 7 可知, 中全新世以来呼伦湖区 $\mathrm{P}_{\mathrm{A}}$ 与 $\mathrm{T}_{\mathrm{C}} 、 \mathrm{~T}_{\mathrm{A}}$ 变化趋势基本一致, 而与 $\mathrm{T}_{\mathrm{W}}$ 变化趋势的关系不是很明显. 同时, $O C B R$ 与湖区 $\mathrm{T}_{\mathrm{C}} 、 \mathrm{~T}_{\mathrm{A}}$ 及 $\mathrm{P}_{\mathrm{A}}$ 大体呈现出 一种反向变化的趋势, 与 $\mathrm{T}_{\mathrm{W}}$ 变化趋势的关系不明显.

相关性分析表明 (表 2), $O C B R$ 与湖区 $\mathrm{T}_{\mathrm{C}} 、 \mathrm{~T}_{\mathrm{A}}$ 及 $\mathrm{P}_{\mathrm{A}}$ 均呈负相关, 即中全新世以来, 呼伦湖 $O C B R$ 随温度升 高及降水量的增加而呈现下降的趋势. 这和我们之前研究得出的近百年来呼伦湖 $O C B R$ 与温度及降水均表现 为正相关的结论 (未发表数据) 相悖, 表明在不同时间尺度上,气候条件对呼伦湖碳埋藏的影响可能是不同的. 


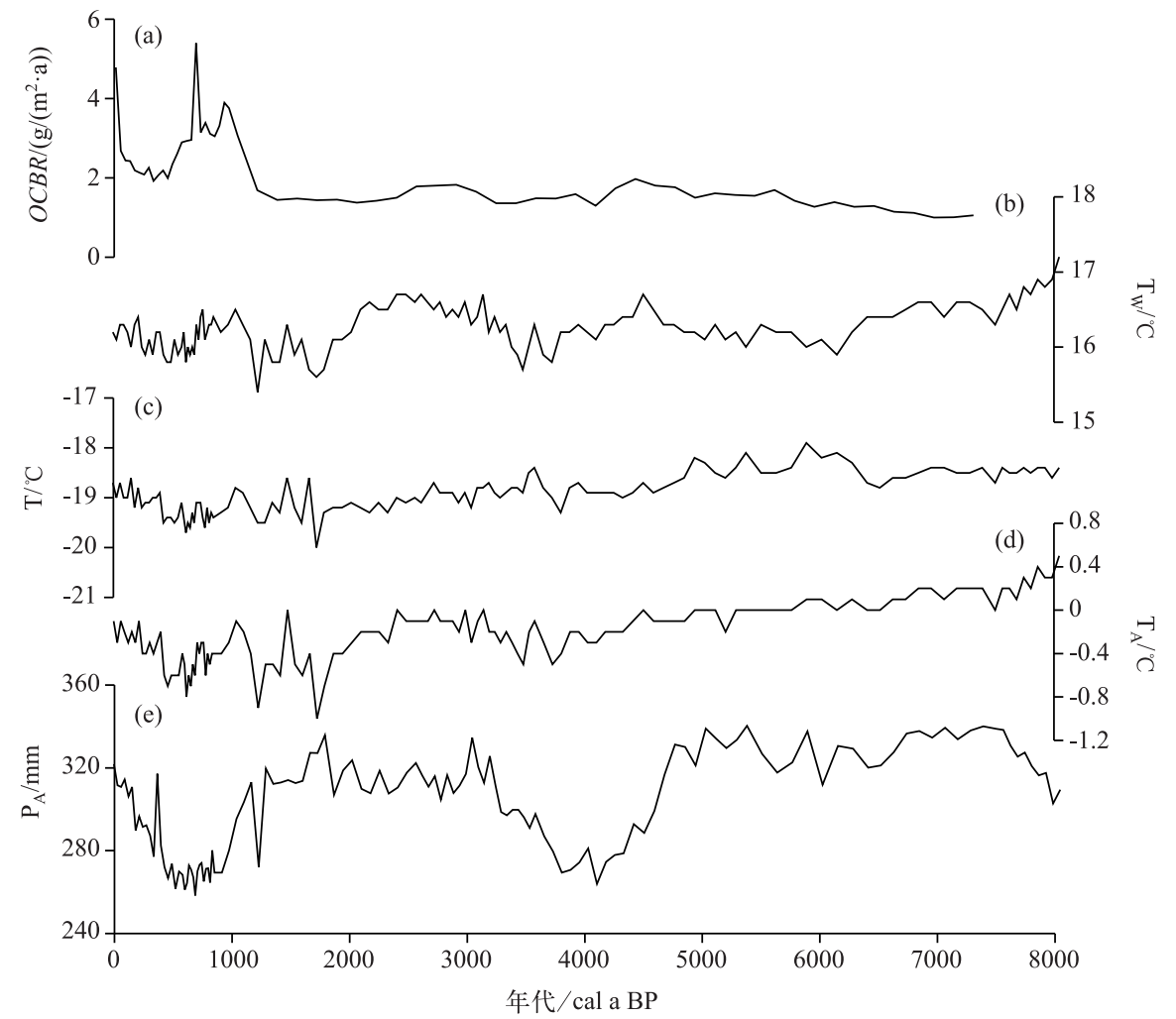

图 7 中全新世以来呼伦湖 $O C B R(\mathrm{a})$ 、湖区 $\mathrm{T}_{\mathrm{W}}(\mathrm{b})^{[42]} 、 \mathrm{~T}_{\mathrm{C}}(\mathrm{c})^{[42]} 、 \mathrm{~T}_{\mathrm{A}}(\mathrm{d})^{[42]}$ 和 $\mathrm{P}_{\mathrm{A}}(\mathrm{e})^{[42]}$ 随时间的变化趋势

Fig.7 Temporal changes of $\operatorname{OCBR}(\mathrm{a}), \mathrm{T}_{\mathrm{W}}(\mathrm{b})^{[42]}, \mathrm{T}_{\mathrm{C}}(\mathrm{c})^{[42]}, \mathrm{T}_{\mathrm{A}}(\mathrm{d})^{[42]}$ and

$$
\mathrm{P}_{\mathrm{A}}(\mathrm{e})^{[42]} \text { since the mid-Holocene in Lake Hulun }
$$

表 2 中全新世以来呼伦湖沉积物 $O C B R$ 与湖区温度、降水之间的相关性分析

Tab.2 Correlation coefficients between OCBR and temperature, precipitation since the mid-Holocene in Lake Hulun

\begin{tabular}{cllccc}
\hline 相关性 & OCBR & $\mathrm{T}_{\mathrm{W}}$ & $\mathrm{T}_{\mathrm{C}}$ & $\mathrm{T}_{\mathrm{A}}$ & $\mathrm{P}_{\mathrm{A}}$ \\
\hline OCBR & 1 & -0.181 & $-0.502^{* *}$ & $-0.315^{*}$ & $-0.502^{* *}$ \\
$\mathrm{~T}_{\mathrm{W}}$ & -0.181 & 1 & $0.372^{*}$ & $0.735^{* *}$ & 0.275 \\
$\mathrm{~T}_{\mathrm{C}}$ & $-0.502^{* *}$ & $0.372^{*}$ & 1 & $0.821^{* *}$ & $0.508^{* *}$ \\
$\mathrm{~T}_{\mathrm{A}}$ & $-0.315^{*}$ & $0.735^{* *}$ & $0.821^{* *}$ & 1 & $0.545^{* *}$ \\
$\mathrm{P}_{\mathrm{A}}$ & $-0.502^{* *}$ & 0.275 & $0.508^{* *}$ & $0.545^{* *}$ & 1 \\
\hline
\end{tabular}

**表示在 0.01 水平 $($ 双侧 $)$ 上显著相关, *表示在 0.05 水平 $($ 双侧 $)$ 上显著相关.

先前研究表明,随降水量增加, 流域植被和土壤碳储量通常也会增加 ${ }^{[43-45]}$, 从而造成湖泊沉积物有机碳 的潜在来源增加. 另外, 流域降水量的增加通常还会造成河流碳储量增加, 从而将更多的河流碳通过地表径 流输人湖泊 ${ }^{[46-48]}$, 导致湖泊沉积速率及 $O C B R$ 增加. 对呼伦湖而言, 该湖地处中温带半干旱区, 降雨量较少, 湖面降水对该湖的影响也相对较小. 随着湖区降水量增加, 湖泊水位升高, 尽管通过径流输人到湖泊的外源 碳增加,但同时湖泊水位升高会导致有机碳在水体的滞留时间变长,进而导致有机碳在沉降至湖底前的矿 化增加及碳埋藏减少, 这可能是呼伦湖 $O C B R$ 与湖区降水量呈负相关的一个重要原因. 此外, 降水对呼伦湖 有机碳埋藏的另外一个可能影响在于, 当湖区降水量下降时, 湖泊水位下降, 导致湖滨、河流沿岸湿地面积 扩大, 在浅水强动力条件下, 可将更多的湖周有机碳带人湖泊, 进而导致呼伦湖外源有机碳输人增加及 
$O C B R$ 增加. 最后, 中全新世以来呼伦湖区温度和降水变化呈较好的正相关关系 $(r=0.434, P<0.01)$, 即降水 量较少的时期往往对应于温度较低的时期, 而低温条件下更有利于有机碳的保存 ${ }^{[39]}$, 这可能也是导致呼伦 湖降水量与 $O C B R$ 显著负相关的重要原因.

值得注意的是,在岩芯顶部, 呼伦湖 $O C B R$ 表现为随降水量升高而呈增加的趋势, 由于时间分辨率较 低, 我们很难给出准确的解释. 初步推测, 我们认为这可能是因为岩芯顶部有机碳埋藏时间较短, 部分有机 碳尚未完全矿化分解, 因而出现有机碳含量及 $O C B R$ 较高的 “假象”. 不同来源的有机碳进入湖泊后不会立 刻也不会全部沉降至沉积物, 其中一部分在异养微生物的矿化分解下转化为 $\mathrm{CO}_{2}$ 和 $\mathrm{CH}_{4}$ 返回至大气圈,一部 分随地表径流输出湖泊, 一部分滞留在湖泊中, 剩余的部分沉降至沉积物中. 其中沉降到沉积物中的有机碳 还会有一部分在微生物的作用下被矿化分解, 以 $\mathrm{CO}_{2}$ 和 $\mathrm{CH}_{4}$ 的形式返回至水体或大气, 余下的未被矿化分解 的部分才会被永久性埋藏. 因此岩芯顶部的有机碳很可能有一部分是由于沉降或者埋藏时间较短, 导致尚 未完全矿化分解, 因此出现顶部有机碳及其埋藏速率较高的现象. 另一个可能的解释为, 近年来呼伦湖湖区 人类活动加强, 导致湖泊逐步富营养化, 导致湖泊初级生产力较高, 内源有机碳含量增加, 因而降水量对呼 伦湖有机碳埋藏的影响实际上可能是叠加了人类活动的共同作用.

温度变化对湖泊碳埋藏的影响相对比较复杂. 总体来说, 主要表现在两个方面:第一,温度升高有利于 湖泊藻类生长, 从而促进湖泊初级生产力增加及自生碳埋藏增加 ${ }^{[8,17-18]}$. 同时, 温度升高会使流域内植物生 长季节变长, 导致流域植被和土壤中的碳储量增加, 进而导致进人湖泊的陆源有机碳潜在来源增加 ${ }^{[8,17-18]}$. 第二, 温度能够影响有机质的矿化速率, 温度增高往往会导致有机碳矿化速率增加, 从而降低湖泊碳埋藏速

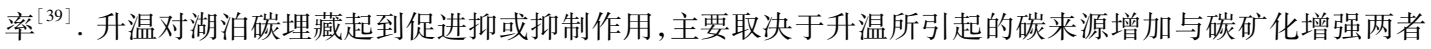
之间的平衡. 如果由温度升高引起的碳来源增加量超过碳的矿化量, 则表现为温度对碳埋藏的促进作用, 反 之, 则表现为抑制作用. 中全新世以来呼伦湖年均温度变化幅度相对较小 ${ }^{[42]}$, 有机碳埋藏与温度之间的负 相关关系可能正是升温所导致的有机碳矿化量超过了有机碳输人的增加量所致. 但这只是我们的一个初步 推测, 具体的内在机制仍待今后进一步深人研究探讨.

致谢: 感谢陶井奎及呼伦湖渔业公司在野外采样中的帮助.

\section{5 参考文献}

[ 1 ] Battin TJ, Luyssaert S, Kaplan LA et al. The boundless carbon cycle. Nature Geoscience, 2009, 2(9) : 598-600.

[ 2 ] Falkowski P, Scholes RJ, Boyle EEA et al. The global carbon cycle: a test of our knowledge of earth as a system. Science, 2000, 290(5490): 291-296.

[ 3 ] Cox PM, Betts RA, Jones CD et al. Acceleration of global warming due to carbon-cycle feedbacks in a coupled climate model. Nature, 2000, 408(6809) : 184-187.

[ 4 ] Alin SR, Johnson TC. Carbon cycling in large lakes of the world: A synthesis of production, burial, and lake-atmosphere exchange estimates. Global Biogeochemical Cycles, 2007, 21(3): 1-12.

[ 5 ] Algesten G, Sobek S, Bergström AK et al. Role of lakes for organic carbon cycling in the boreal zone. Global Change Biology, 2004, 10(1): 141-147.

[ 6 ] Cole JJ, Prairie YT, Caraco NF et al. Plumbing the global carbon cycle: integrating inland waters into the terrestrial carbon budget. Ecosystems, 2007, 10(1): 172-185.

[ 7 ] Downing JA, Cole JJ, Middelburg JJ et al. Sediment organic carbon burial in agriculturally eutrophic impoundments over the last century. Global Biogeochemical Cycles, 2008, 22(1): 1-10.

[8] Heathcote AJ, Downing JA. Impacts of eutrophication on carbon burial in freshwater lakes in an intensively agricultural landscape. Ecosystems, 2012, 15(1): 60-70.

[ 9 ] Tranvik LJ, Downing JA, Cotner JB et al. Lakes and reservoirs as regulators of carbon cycling and climate. Limnology and Oceanography, 2009, 54(6part2) : 2298-2314.

[10] Anderson NJ, D'andrea W, Fritz SC. Holocene carbon burial by lakes in SW Greenland. Global Change Biology, 2009,15 (11) : 2590-2598.

[11] Campbell ID, Campbell C, Vitt DH et al. A first estimate of organic carbon storage in Holocene lake sediments in Alberta, Canada. Journal of Paleolimnology, 2000, 24(4) : 395-400. 
[12] Ferland ME, Giorgio PA, Teodoru CR et al. Long-term C accumulation and total C stocks in boreal lakes in northern Québec. Global Biogeochemical Cycles, 2012, 26(4):1-10.

[13] Kastowski M, Hinderer M, Vecsei A. Long-term carbon burial in European lakes: Analysis and estimate. Global Biogeochemical Cycles, 2011, 25(3): 1-12.

[14] Kortelainen P, Pajunen H, Rantakari M et al. A large carbon pool and small sink in boreal Holocene lake sediments. Global Change Biology, 2004, 10(10): 1648-1653.

[15] Wang M, Chen H, Yu Z et al. Carbon accumulation and sequestration of lakes in China during the Holocene. Global Change Biology, 2015, 21(12): 4436-4448.

[16] Gui ZF, Xue B, Yao SC et al. Organic carbon burial in lake sediments in the middle and lower reaches of the Yangtze River Basin, China. Hydrobiologia, 2013, 710(1): 143-156.

[17] Dong XH, Anderson NJ, Yang XD et al. Carbon burial by shallow lakes on the Yangtze floodplain and its relevance to regional carbon sequestration. Global Change Biology, 2012, 18(7) : 2205-2217.

[18 ] Xu H, Lan JH, Liu B et al. Modern carbon burial in Lake Qinghai, China. Applied Geochemistry, 2013, 39: 150-155.

[19] Yu ZT, Wang XJ, Zhao CY et al. Carbon burial in Bosten Lake over the past century: Impacts of climate change and human activity. Chemical Geology, 2015, 419: 132-141.

[20] Yang H, Xing YP, Xie P et al. Carbon source/sink function of a subtropical, eutrophic lake determined from an overall mass balance and a gas exchange and carbon burial balance. Environmental Pollution, 2008, 151(3) : 559-568.

[21] Duan XN, Wang XK, Lu F et al. Carbon sequestration and its potential by wetland ecosystems in China. Acta Ecologica Sinica, 2008, 2(28) : 463-469. [段晓男, 王效科, 逯非等. 中国湿地生态系统固碳现状和潜力. 生态学报, 2008, 2(28): 463-469.]

[22] Wen R, Xiao J, Chang Z et al. Holocene climate changes in the mid-high-latitude-monsoon margin reflected by the pollen record from Hulun Lake, northeastern Inner Mongolia. Quaternary Research, 2010, 73(2) : 293-303.

[23] Hulun Lake Fishery Co., Ltd ed. Annals of Hulun Lake. Hohhot: Inner Mongolia Culture Press, 2008: 18-35. [呼伦湖渔 业有限公司编. 呼伦湖志. 呼和浩特: 内蒙古文化出版社, 2008: 18-35.]

[24] Kiyokazu K, Cheng Y. Floristic composition, grazing effects and above-ground plant biomass in the Hulunbeier Grasslands of Inner Mongolia, China. Journal of Ecology and Environment, 2008, 31(4) : 297-307.

[25] Jiang ZF, Li CY, Zhang S et al. Phytoplankton and nutrition evaluation in Hulun Lake. Journal of Agro-Environment Science, 2011, 30(4): 726-732. [姜忠峰, 李畅游, 张生等. 呼伦湖浮游植物调查与营养状况评价. 农业环境科学学 报, 2011, 30(4): 726-732.]

[26] Reimer PJ, Bard E, Bayliss A et al. IntCal13 and Marine13 radiocarbon age calibration curves 0-50,000 years cal BP. 2013. University of Arizona, Radiocarbon, 2013, 55(4) : 1869-1887.

[27] Nakamura T, Niu E, Oda H et al. The HVEE tandetron AMS system at Nagoya University. Nuclear Instruments and Methods in Physics Research Section B: Beam Interactions with Materials and Atoms, 2000, 172(1) : 52-57.

[28] Xiao JL, Chang ZG, Wen RL et al. Holocene weak monsoon intervals indicated by low lake levels at Hulun Lake in the monsoonal margin region of northeastern Inner Mongolia, China. The Holocene, 2009, 19(6) : 899-908.

[29] Gui ZF. The carbon burial of lake sediments in eastern China over the past 100 years[Dissertation]. Nanjing: Nanjing Institute of Geography and Limnology, Chinese Academy of Sciences, 2012. [桂智凡. 近百年来中国东部地区湖泊沉积 物碳埋藏研究 [学位论文]. 南京: 中国科学院南京地理与湖泊研究所, 2012.]

[30] Shen J, Wang SM, Yang XD. Measurement of organic carbon stable isotope in lacustrine sediments and its significance on paleoclimate and environment. Oceanologia et Limnologia Sinica , 1996, 27(4) : 400-404. [沈吉, 王苏民，羊向东. 湖 泊沉积物中有机碳稳定同位素测定及其古气候环境意义. 海洋与湖沼, 1996, 27(4) : 400-404.]

[31] Meyers PA. Organic geochemical proxies of paleoceanographic, paleolimnologic, and paleoclimatic processes. Organic Geochemistry, 1997, 27(5): 213-250.

[32] Chen X, Chuai X, Yang L et al. Climatic warming and overgrazing induced the high concentration of organic matter in Lake Hulun, a large shallow eutrophic steppe lake in northern China. Science of the Total Environment, 2012, 431: 332-338.

[33] Schubert CJ, Calvert SE. Nitrogen and carbon isotopic composition of marine and terrestrial organic matter in Arctic Ocean sediments : implications for nutrient utilization and organic matter composition. Deep Sea Research Part I: Oceanographic 
Research Papers, 2001, 48(3) : 789-810.

[34] Shen J, Xue B, Wu JL et al eds. Lake sediments and environment changes. Beijing: Science Press, 2010: 220-221. [沈 吉, 薛滨, 吴敬禄等. 湖泊沉积与环境演化. 北京: 科学出版社, 2010: 220-221.]

[35] Smith BN, Epstein S. Two categories of ${ }^{13} \mathrm{C} /{ }^{12} \mathrm{C}$ ratios for higher plants. Plant Physiology, 1971, 47(3) : 380-384.

[36] Qian JL, Wang SM, Xue B et al. A method of quantitative estimating terrestrial organic carbon in lake sedimentation research. Chinese Science Bulletin, 1997, 42(15): 1655-1658. [ 钱君龙, 王苏民, 薛滨等. 湖泊沉积研究中的一种定量 估算陆源有机碳的方法. 科学通报, 1997, 42(15): 1655-1658.]

[37] Zhang FJ, Xue B, Yao SC et al. The Holocene megathermal organic carbon burial rates in Chinese lake sediments. Quaternary Sciences, 2013, 33(2) : 401-402. [张风菊, 薛滨, 姚书春等. 全新世大暖期中国湖泊碳埋藏速率初步研究. 第 四纪研究, 2013, 33(2) : 401-402.]

[38] Squires MM, Mazzucchi D, Devito KJ. Carbon burial and infill rates in small western Boreal lakes: physical factors affecting carbon storage. Canadian Journal of Fisheries and Aquatic Sciences, 2006, 63(4) : 711-720.

[39] Gudasz C, Bastviken D, Steger K et al. Temperature-controlled organic carbon mineralization in lake sediments. Nature, 2010, 466(7305): 478-481.

[40] Anderson NJ, Dietz RD, Engstrom DR. Land-use change, not climate, controls organic carbon burial in lakes. Proceedings of the Royal Society of London B: Biological Sciences, 2013, 280(1769) : 1-7.

[41] Brothers SM, Hilt S, Attermeyer K et al. A regime shift from macrophyte to phytoplankton dominance enhances carbon burial in a shallow, eutrophic lake. Ecosphere, 2013, 4(11): 1-17.

[42] Wen RL, Xiao JL, Chang ZG et al. Holocene precipitation and temperature variations in the East Asian monsoonal margin from pollen data from Hulun Lake in northeastern Inner Mongolia, China. Boreas, 2010, 39(2) : 262-272.

[43] Hontoria C, Saa A, Rodríguez-Murillo JC. Relationships between soil organic carbon and site characteristics in peninsular Spain. Soil Science Society of America Journal, 1999, 63(3): 614-621.

[44] Post WM, Emanuel WR, Zinke PJ et al. Soil carbon pools and world life zones. Nature, 1982, 298(5870) : 156-159.

[45] Lan J, Xu H, Liu B et al. A large carbon pool in lake sediments over the arid/semiarid region, NW China. Chinese Journal of Geochemistry, 2015, 34(3) : 289-298.

[46] Andersson T, Nilsson A, Jansson M eds. Coloured substances in Swedish lakes and rivers-temporal variation and regulating factors. Humic substances in the aquatic and terrestrial environment. Berlin: Springer, 1991: 243-253.

[47] Erlandsson M, Buffam I, Fölster J et al. Thirty-five years of synchrony in the organic matter concentrations of Swedish rivers explained by variation in flow and sulphate. Global Change Biology, 2008, 14(5) : 1191-1198.

[48] Schindler DE, Carpenter SR, Cole JJ et al. Influence of food web structure on carbon exchange between lakes and the atmosphere. Science, 1997, 277(5323) : 248-251. 